\title{
Coordination d'Activités dans les Chaînes Logistiques: une Approche Multi-Agents par Formation de Coalitions
}

\author{
Dhouha Anane* — Samir Aknine** — Suzanne Pinson * \\ * Université Paris-Dauphine \\ CNRS/LAMSADE UMR 7042 \\ 75775 Paris cedex 16, France \\ dhouhaanane@yahoo.fr, suzanne.pinson@.dauphine.fr \\ ** Université Paris 6 \\ Lip6 104 Avenue du Président Kennedy \\ 75016, Paris, France \\ Samir.aknine@lip6.fr
}

\begin{abstract}
RÉSUMÉ. Les entreprises évoluent dans un environnement de plus en plus compétitif et exigeant en termes de flexibilité et de réactivité. L'utilisation des modèles issus de l'Intelligence Artificielle Distribuée (IAD) et plus particulièrement des systèmes multi-agents (SMA) dans les outils de gestion des entreprises s'avère être efficace pour simuler et reproduire les comportements collaboratifs et adaptatifs tels qu'ils apparaissent actuellement dans les entreprises. Cet article modélise la coordination des différentes parties collaboratives aussi bien internes qu'externes d'une chaîne logistique en utilisant les modèles de coordination par formation de coalitions proposés dans les SMA. Dans une première partie, nous faisons un tour d'horizon des travaux déjà effectués sur ce problème. Dans une deuxième partie, nous proposons une modélisation agent, un algorithme de formation de coalitions ainsi qu'un protocole d'interaction entre agents indispensable pour mettre en ceuvre cette coordination distribuée. Finalement, nous illustrons notre démarche par un exemple pris dans le domaine de l'industrie avionique.
\end{abstract}

\begin{abstract}
Several factors have meant that enterprises operate in an environment increasingly demanding in terms of flexibility and reactivity. The introduction of the entities resulting from Distributed Artificial Intelligence (DAI) and Multi-Agent Systems (MAS) in the management of enterprises prove to be an interesting technology to simulate and reproduce the collaborative and adaptive behavior of enterprises. This article models the coordination of the various collaborative parties both inside and outside a supply chain using coordination methods found in MAS i.e. coalition formation mechanisms. The first part of this paper proposes a state of the art. In the second part, we present our agent modeling, and then we detail the coalition formation algorithm as well as the interaction protocol between agents used by the distributed coordination algorithm. Lastly, we illustrate our propositions with an example chosen in the industrial domain.

MOTS-CLÉS: Châ̂nes Logistiques, Multi Agents, Formation de coalitions, coordination.

KEYWORDS: Supply Chains, Multi-Agent Systems, Coalitions formation, Coordination.
\end{abstract}




\section{Introduction}

Par définition, une chaîne logistique d'une entreprise est un ensemble d'entités autonomes, internes ou externes à l'entreprise, interagissant les unes avec les autres dans le but de maximiser un bien-être global en recherchant un compromis par rapport à leurs propres contraintes et buts individuels. Ce compromis n'est pas toujours simple à atteindre étant donné que l'entreprise évolue dans un environnement constamment changeant et dynamique. Les caractéristiques de tels systèmes, à savoir complexité, environnement dynamique, autonomie de chaque entité intervenant dans la chaîne, incitent les chercheurs à utiliser l'approche multiagents pour les modéliser et en étudier le comportement. Les travaux les plus connus dans le domaine sont ceux de (Fox et al. 1996, 2000).

L'objectif de notre travail est de proposer une méthode de coordination par formation de coalitions pour modéliser les chaînes logistiques dans leur globalité. Selon (Shehory et Kraus, 1998), une Coalition se définit comme un groupe d'agents qui décident de coopérer afin de réaliser un but commun. A ce but est associée une valeur (utilité) partagée entre les différents agents formant la coalition. Nous cherchons ainsi à modéliser les relations de partenariats entre les différentes entités tout en distinguant les coalitions selon deux niveaux d'abstraction :

- Coalitions internes : ce sont les coalitions qui se forment entre les différentes entités à l'intérieur de la même chaîne comme par exemple le regroupement de plusieurs usines de la même entreprise afin de faire face à une importante commande.

- Coalitions externes : ce sont les partenariats qui peuvent s'établir entre des entités de la chaîne et des entités externes à l'entreprise et ce dans le but de se procurer des ressources qui leur manquent ou des compétences dont elles ne disposent pas.

Cet article est organisé comme suit: la section 2 présente une revue des approches existantes. Dans les sections 3 et 4 nous décrivons le problème de coordination par un exemple puis nous le formalisons par une approche multiagents. La section 5 propose une méthode de formation de coalitions adaptée à ce problème de coordination distribuée en présentant un algorithme ainsi que le protocole d'interaction nécessaire à sa mise en œuvre. Enfin nous concluons sur ce travail.

\section{Etat de l'art}

La modélisation de la gestion et de l'optimisation des chaînes logistiques (CL) est l'un des domaines privilégiés de la Recherche Opérationnelle (RO) (Kok et al. 2003), (Beamon, 1998), (Giard et Balin, 2007) : localisation d'usines, planification de la production, gestion de stocks, transports et distribution. La plupart de ces travaux proposent des approches centralisées basées sur des modèles analytiques. Depuis l'émergence des techniques distribuées et des systèmes multi-agents, plusieurs approches simulent les comportements collaboratifs entre les entités 
composant une chaîne logistique (Dodd et al., 2001). L'avantage de ces approches consiste en la possibilité de représenter explicitement le comportement des entités ainsi que leurs interactions et leur organisation (Parunak, 1996), (Parunak et al., 1998).

(Swaminathan et al., 1998) modélisent la dynamique des chaînes logistiques par une approche multi-agents. L'apport de leur travail est la combinaison des méthodes analytiques et des méthodes de simulation afin de pouvoir modéliser respectivement l'aspect statique et l'aspect dynamique des chaînes logistiques.

D'autres travaux se sont orientés vers l'étude de la dynamique des chaînes logistiques autrement dit la coordination en utilisant des techniques plus spécifiques aux SMA comme les langages et les protocoles d'interaction. Parmi ces travaux, (Fox et Barbuceanu, 1996) définissent un système de coordination à travers des conversations structurées tout en introduisant un langage de communication (COOL) qui se base sur les principes du langage $\mathrm{KQML}^{1}$ et les automates à états finis. Ce travail a été détaillé dans (Fox et al., 2000) et propose une architecture logicielle par le biais d'un outil de construction orientée agent (Agent Building Shell «BS »).

D'autres outils de modélisation ont été utilisés pour décrire la coordination entre les différents agents d'une chaîne logistique comme le langage AUML ${ }^{2}$ (Huget, 2002), (Nfaoui et al. 2006) afin de représenter les différents protocoles de négociation entre agents.

D'autres travaux ne modélisent qu'une partie de la chaîne logistique (clients) comme ceux réalisés par (Chaib-draa et al., 2002) qui utilisent les méthodes de formation de coalitions pour regrouper plusieurs acheteurs afin de diminuer les coûts ou ceux réalisés par (Hahndel et al., 1994) qui utilisent un protocole de négociation pour coordonner la planification des activités de production. A ce jour, peu de travaux existent sur l'utilisation de la méthode de formation de coalitions pour modéliser la coordination dans les chaînes logistiques.

\section{Illustration du problème}

Nous considérons une entreprise qui fabrique et commercialise des avions. Nous avons choisi ce domaine industriel en raison de la complexité de la chaîne logistique qui met en œuvre principalement la sous-traitance de plusieurs pièces et la multitude d'entités qui y contribuent. Cette entreprise est localisée principalement en France mais elle possède plusieurs sites de production (usines) sur plusieurs continents. Elle possède également divers centres de distribution en Europe qui livrent différents marchés (Europe, Moyen-0rient ...). Par ailleurs, ses fournisseurs (notés F-P dans la figure 1) sont physiquement dispersés.

1. KQML : Knowledge Query and Manipulation Language

2. AUML : Agent Unified Modeling Language 
A l'intérieur de chaque usine (cf. figure 1), nous pouvons identifier plusieurs autres unités qui travaillent en étroite collaboration les unes avec les autres. Ce qui complique encore une telle situation ce sont les liens qui existent entre ces entités et les entités des autres chaînes logistiques, d'où le retard ou disfonctionnement au niveau d'un point de la chaîne qui peut se propager au niveau de tous les points qui lui sont reliés.

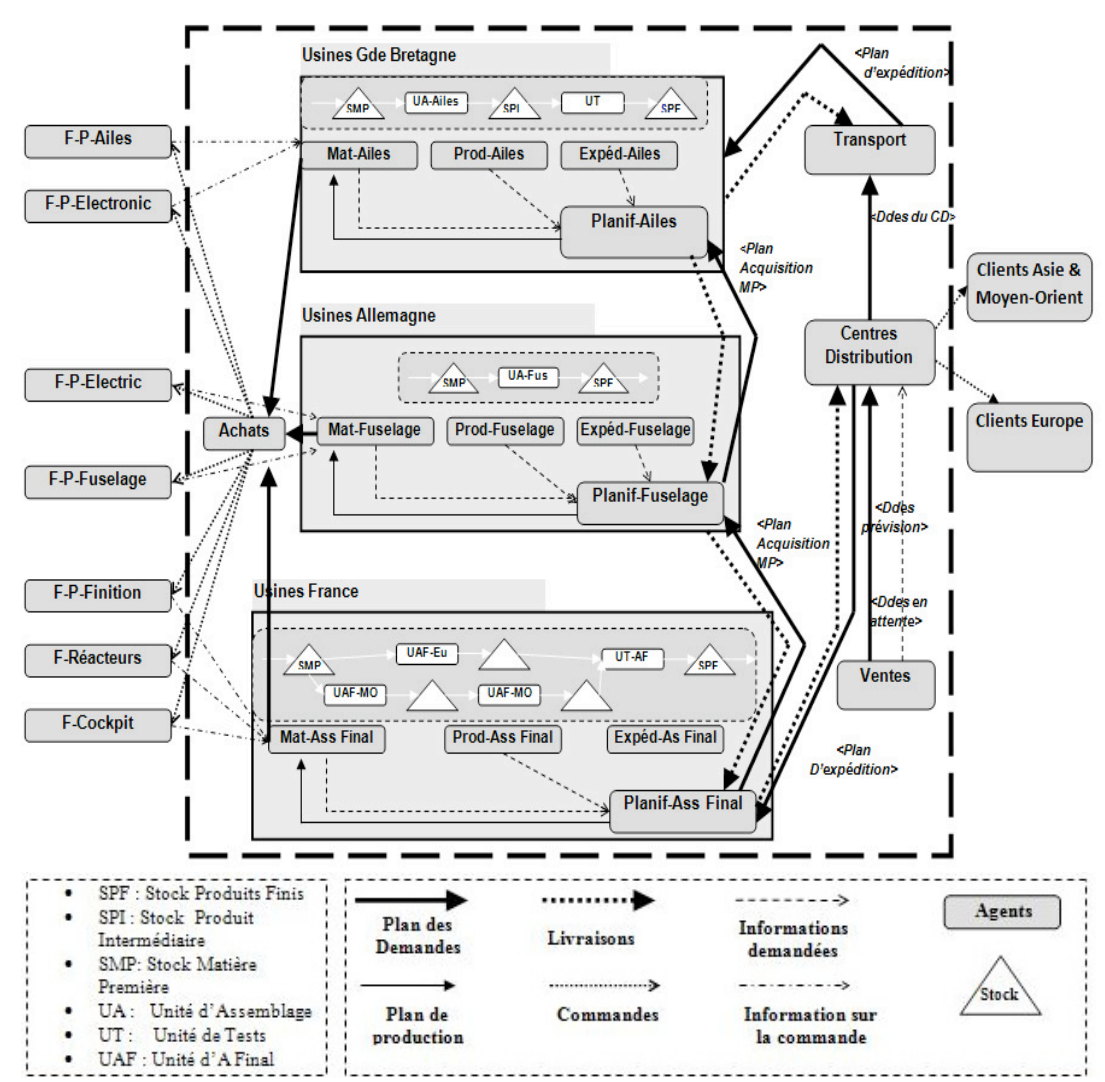

Figure 1. Exemple d'une chaîne logistique et des interactions entre les entités

\section{Modélisation multi-agents du problème}

\subsection{Identification des agents}

Dans notre approche agent, nous proposons de modéliser chacune des entités composant la chaîne logistique par un agent. Nous distinguons deux niveaux d'abstraction entre agents internes et externes: 
- Les agents internes : ce sont les agents situés à l'intérieur de l'entreprise. Dans cette catégorie, nous distinguons deux niveaux : niveau entreprise et niveau usine.

- Agents au niveau de l'usine :

- Agents 'Planif' : ces agents s'occupent de la planification de la production en se basant sur les plans des demandes reçues et sur les communications avec les autres agents de l'usine.

- Agents 'Produc' : ces agents gèrent la production et les stocks des produits intermédiaires. Ces agents possèdent une parfaite connaissance de la disposition de l'usine en terme de ressources, de machines et de leurs emplacements...

- Agents 'Expéd' : ces agents gèrent les produits finis de chaque usine et de leurs expéditions.

- Agents 'Mat' : ces agents gèrent les stocks des matières premières (SMP) et traitent toutes les données relatives aux commandes et réception de matières premières.

- Agents 'Info' : ces agents traitent toutes les informations relatives au bon fonctionnement de l'usine (capacités et pannes des machines, données sur les stocks SMP, SPI et SPF)

- Agents au niveau de l'entreprise :

- Agent 'Achat' : cet agent représente la fonction achat de l'entreprise incluant la fonction de sélection et de communication avec les fournisseurs.

- Agent 'Vente' : cet agent regroupe les fonctions de marketing et de commercialisation des produits de l'entreprise. Il assure les prévisions de la demande en établissant des communications avec les clients.

- Agent 'Transport' : cet agent assure le déplacement des produits entre les usines et les centres de distribution.

- Agents 'CD' : ces agents représentent les différents centres de distribution de l'entreprise.

- Agents 'Logistic' : ces agents assurent la bonne communication entre les différents agents (achat, vente, expéd, planif et prod). Ils gèrent aussi les événements qui peuvent surgir sur le système comme par exemple la modification d'une commande par un client, la rupture de stock d'un des produits, le changement d'un fournisseur...

- Les agents externes : ce sont les agents situés à l'extérieur de l'entreprise.

- Agents 'Client' : ces agents définissent les clients de l'entreprise. Dans notre exemple nous avons identifié deux agents représentant chacun un marché : le marché européen, le marché asiatique et Moyen-Orient.

- Agents 'Fournisseur' : chaque fournisseur est représenté par un agent, dans notre exemple nous avons recensé 7 fournisseurs.

- Agents 'Part-econ': cet agent représente toute autre entité dans l'environnement susceptible d'avoir une relation avec l'entreprise (concurrents, partenaires de transport, ...). 
Comme nous l'avons vu précédemment, dans les chaînes logistiques, les différentes entités représentées par des agents doivent se coordonner pour réaliser des tâches communes. Dans ce qui suit, nous nous appuyons sur les travaux de (Shehory et Kraus, 1996, 1998) et de (Aknine et al. 2004) pour proposer une nouvelle approche de formation de coalitions entre agents autonomes qui prend en compte deux niveaux d'abstraction à savoir des coalitions internes et des coalitions externes et qui utilise les notions de priorité entre tâches et de préférence d'un agent par rapport à un autre.

\section{Coordination dans les Chaînes Logistiques par formation de coalitions}

\subsection{Définition des concepts}

Une chaîne de tâches est représentée par un graphe orienté acyclique $\langle T C, E\rangle$, tel que $T C=\left\{t_{1} \cdots t_{m}\right\}$ représente l'ensemble des tâches complexes pour lesquelles un agent peut éventuellement lancer un processus de formation de coalitions afin de les réaliser. $E=\left\{\left(t_{i}, t_{j}\right)\right\}$ est l'ensemble des arcs reliant la tâche $t_{i}$ qui précède la tâche $t_{j}$

avec $\left(\varnothing, t_{1}\right) \in E$ comme tâche de début, et $\left(t_{m}, \varnothing\right) \in E$ comme tâche de fin. Nous définissons, à présent, les concepts suivants :

- Soit $A=\left\{a_{1} \cdots a_{n}\right\}$ l'ensemble de tous les agents du système. Cet ensemble est formé de deux sous ensembles $A=A^{I} \cup A^{E}$ comprenant respectivement les agents internes $A^{I}=\left\{a_{1} \cdots a_{k}\right\}$ et les agents externes $A^{E}=\left\{a_{k+1} \cdots a_{n}\right\}$.

- Soit $T=\left\{t_{1} \cdots t_{c}\right\}$ l'ensemble des tâches de la chaîne tel que $T C \subseteq T$.

- Pour la formation de coalitions, un agent détermine, selon un modèle de préférences, un ensemble d'agents potentiels noté $A P$ incluant lui-même.

- Après la formation de coalitions, on note par $A R$ l'ensemble des agents de la chaîne ne participant pas à des coalitions mais contribuant à l'une des activités de cette chaîne.

- Chaque agent $a_{i}$ a un vecteur de capacités noté $Q=\left\langle\dot{q}_{1} \cdot \cdot \dot{q}_{p}\right\rangle$.

- Chaque activité ou tâche complexe $t_{s}$ a un coût d'exécution noté $c_{t_{s}}$ qui n'est autre que l'agrégation des différents coûts des agents contribuant à son exécution. Ce coût représente la fonction d'utilité de l'agent. Nous supposons que cette fonction d'utilité est définie par une fonction linéaire.

- On définit aussi, $c p_{a_{i}, a_{j}}^{t_{s}}$, le coût de passation du résultat de l'activité $t_{s}$ de l'agent $a_{i}$ à l'agent $a_{j}$, mais ce paramètre sert à optimiser le coût total de la chaîne. 
L'objectif de tout agent est de minimiser son coût individuel et par conséquent de minimiser le coût de toute la chaîne. Dans le cas d'une coalition de plusieurs agents et afin de déterminer le coût d'une activité, une variable binaire est utilisée :

$$
Z_{t_{s}}^{a_{i}}= \begin{cases}1 & \text { si l'agent } a_{i} \text { est séléctionné pour contribuer à la tâche } t_{s} \\ 0 & \text { Sinon }\end{cases}
$$

Avec $a_{i} \in A P$ et $t_{s} \in T C$

Le but du système revient à réduire le coût total C.T en résolvant le problème suivant :

$$
\begin{gathered}
\text { Min } C . T=\sum_{a_{i} \in A P P} \sum_{t_{s} \in T C} z_{t_{s}}^{a_{i}} c_{t_{s}}^{a_{i}}+\sum_{\left(t_{s}, t_{l}\right) \in E} \sum_{a_{i}, a_{j} \in A P} z_{t_{s}}^{a_{i}} z_{t_{l}}^{a_{j}} c p_{a_{i} a_{j}}^{t_{s}}+\sum_{a_{i} \in A R} \sum_{t_{s} \in T} c_{t_{s}}^{a_{i}} \\
\text { s/c: } \quad \forall t_{m}, t_{l} \in T C,\left(t_{m}, t_{l}\right) \in E \\
\sum_{t_{s} \in T} q_{t_{s}}^{a_{i}} z_{t_{s}}^{a_{i}} \leq Q^{i}
\end{gathered}
$$

(a) pour satisfaire la contrainte d'ordre entre les tâches.

(b) La somme des capacités de l'agent $a_{i}$ investies dans toutes les tâches ne doit pas dépasser sa capacité totale.

Les deux premières parties de [1] représentent le coût des activités pour lesquelles des coalitions sont formées. La troisième partie décrit le coût du reste des activités de la chaîne. Nous définissons la préférence d'un agent par rapport à un autre par la matrice de préférences suivante :

$$
\prod_{a_{i}}^{p}=\left(\begin{array}{cccc}
y_{i 1}^{1} & y_{i 2}^{1} & \ldots & y_{i n}^{1} \\
y_{i 1}^{2} & y_{i 2}^{2} & \ldots & y_{i n}^{2} \\
y_{i 1}^{p} & y_{i 2}^{p} & \ldots & y_{i n}^{p}
\end{array}\right) \text { avec }\left\{\begin{array}{l}
i \text { l'indice des agents } \in N \\
p \text { l'indice des critères }
\end{array}\right.
$$

Chaque élément $y_{i j}^{k}$ de la matrice représente la préférence d'un agent $a_{i}$ pour l'agent $a_{j}$ selon le critère $k$. Pour obtenir la préférence multicritère d'un agent pour un autre, cette matrice est réduite à un vecteur à l'aide d'un opérateur d'agrégation qui peut être la somme pondérée ou l'intégrale de Choquet (Grabisch, 1996). Nous avons choisi d'utiliser le deuxième opérateur qui a l'avantage, contrairement à la somme pondérée, de ne pas biaiser le résultat de l'agrégation lorsque les critères ne sont pas indépendants. Il se base sur le principe suivant : l'agent $a_{i}$ classe par ordre décroissant la colonne de ses préférences pour un agent $a_{j}$ selon des critères spécifiques $y_{i j}^{k}$, étant donné les poids individuels et collectifs des différents critères $k$ notés $\mu\left(E_{k}\right)$ définis par le concepteur du système (avec $E_{k}$ un ensemble composé 
d'un seul ou de plusieurs critères). Il calcule ensuite le vecteur de ses préférences

$\prod_{a_{i}}=\left(x_{i j}\right)$ comme suit :

$$
x_{i j}=\sum_{0 \leq k \leq p}\left(y_{i j}^{k}-y_{i j}^{k+1}\right) \mu\left(E_{k}\right) \text { Avec } y_{i j}^{p+1}=0 \text { et } y_{i i}^{k}=0
$$

Nous définissons un autre concept: l'indice d'attraction qui peut être unilatérale (U-Att) ou bilatérale (B-Att) :

- L'attraction unilatérale représente une mesure de la force qui permet à un agent de convaincre les autres agents, pas encore membres, de rejoindre la coalition. Pour pouvoir la déterminer, l'agent classe les différentes valeurs du vecteur

$\prod_{a_{i}}=\left(x_{i j}\right)$ selon un ordre décroissant. Formellement, cette valeur est définie comme suit :

$$
\text { U-Att }\left(\boldsymbol{a}_{i}\right)=\sum_{j=1}^{n}\left(x_{i, j}-x_{i, j+1}\right) \mu\left(A_{j}\right) \text { avec } i \neq j
$$

Dans [3], $\mu\left(A_{j}\right)$ étant un poids prédéfini pour l'agent $a_{i}$ ou pour l'ensemble d'agent $A_{j} \in A=\left\{a_{1} \ldots a_{n}\right\}$

- L'attraction bilatérale représente à la fois le désir d'un agent de rejoindre une coalition et le désir de cette coalition d'intégrer cet agent. Elle est définie comme suit :

$$
\text { B-Att }\left(a_{i}, C_{k}\right)=\prod_{k i} \times \Re\left(a_{i}, C_{k}\right)
$$

Dans [4], $\Re\left(a_{i}, C_{k}\right)$ représente la préférence d'un agent $a_{i}$ pour la coalition $C_{k}$ et $\prod_{k i}$ représente le modèle de préférence d'une coalition $C_{k}$ pour l'agent $a_{i}$.

\subsection{Principe du mécanisme et algorithmes associés}

Un agent $a_{i}$ reçoit un nouveau message provenant de son environnement, l'analyse à travers sa fonction de détection qui le transmet à la fonction de décision. Sa fonction de décision, en consultant les objectifs de l'agent, l'état actuel et ses connaissances, choisit quelle action entreprendre. La fonction d'action est alors activée pour lancer le processus de coordination en envoyant des messages aux autres agents, elle met à jour l'état et les connaissances de l'agent. S'il s'agit d'un 
processus de formation de coalitions afin d'exécuter une tâche complexe l'agent $a_{i}$ se met dans le rôle d'initiateur et entre en communication avec les autres agents selon l'algorithme suivant dont les deux étapes principales sont décrites ci-dessous. Le protocole que nous proposons repose sur le principe d'appel d'offres des protocoles classiques par exemple le «Contract Net Protocol » (Davis et Smith, 1988). En revanche, son objectif est différent puisque nous recherchons l'élaboration d'accords multi - latéraux entre plusieurs d'où une spécification différente de ce protocole.

\section{- Etape 1 :}

L'agent, après avoir construit son modèle de préférence, contacte les agents internes et externes qu'il préfère c.-à-d. ayant les valeurs maximales dans son modèle de préférences (cf. figure 2 ). Il est à noter qu'on se limite au modèle de préférence d'un agent $a_{i}$ pour un agent $a_{j}$ selon différents critères prédéfinis auparavant par le concepteur du système comme par exemple le délai, la distance, la qualité...

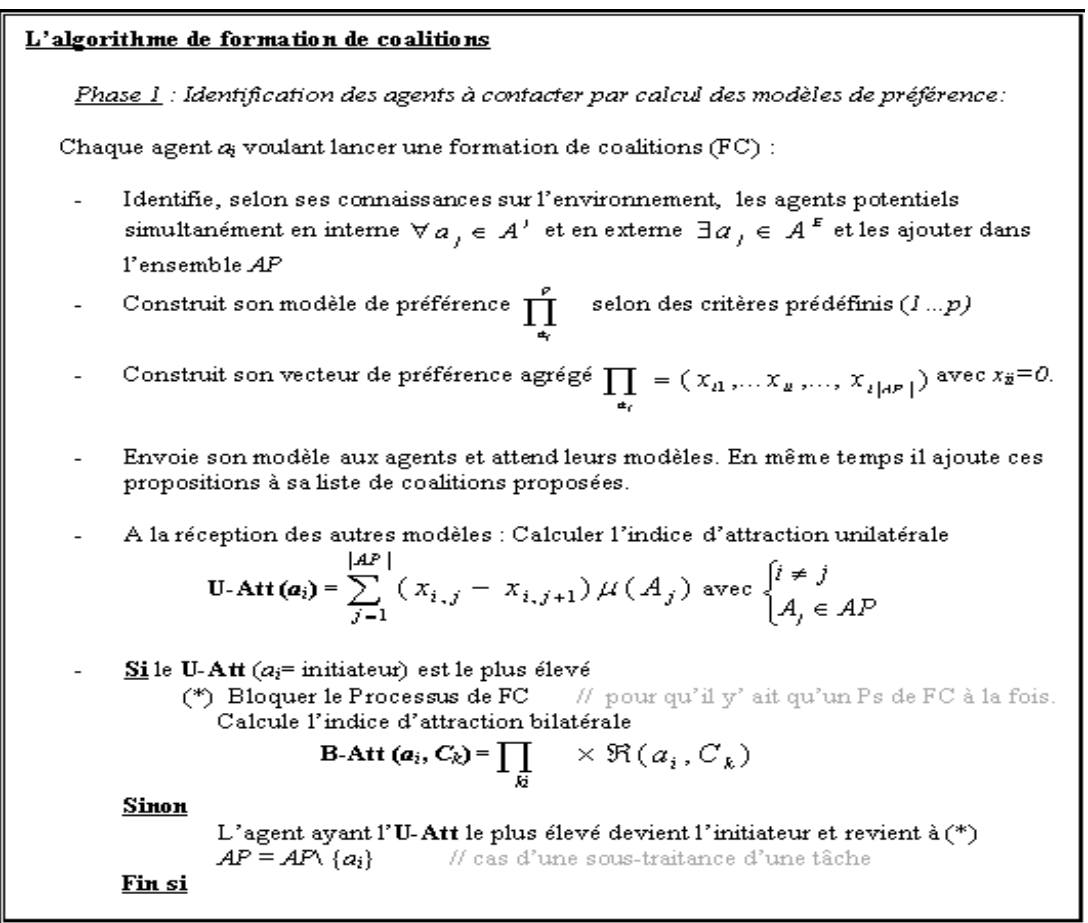

Figure 2. Phase 1 de l'algorithme de formation de coalitions 


\section{- Etape 2 :}

Les agents sollicités consultent leurs capacités pour déterminer s'ils peuvent contribuer à cette activité en examinant la liste des activités qui la précèdent comme par exemple les ressources appropriées, la quantité de matières premières nécessaires...; Ensuite ils répondent à l'agent initiateur par une acceptation temporaire ou un refus. Si l'agent initiateur reçoit un refus lui seul peut décider s'il continue le processus ou l'annule (cf. figure 3). Dans le premier cas, il envoie un message aux agents pour qu'ils confirment leurs participations et donc s'ils acceptent définitivement, l'agent initiateur les rajoute à la liste de coalitions confirmées et détermine le coût total.

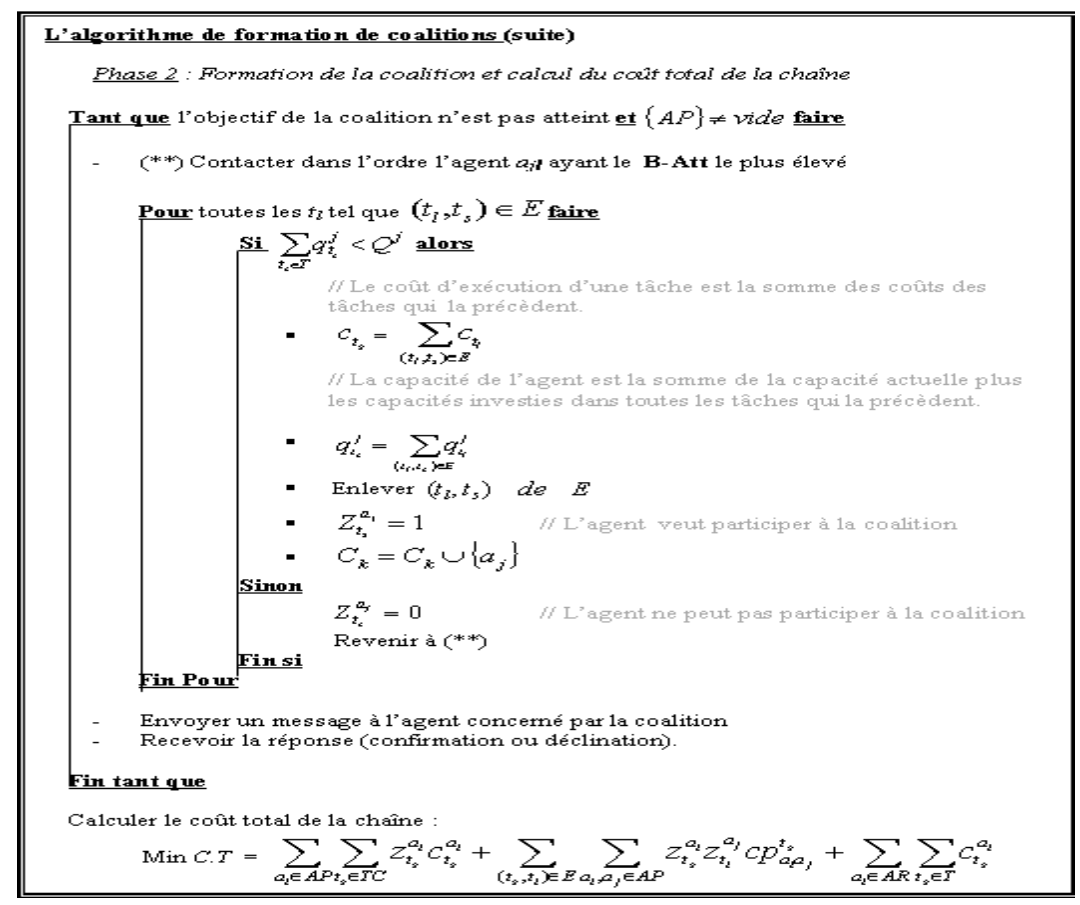

Figure 3. Phase 2 de l'algorithme de formation de coalitions

\subsection{Illustration de l'exemple}

A présent, revenons à notre exemple de la chaîne logistique de la compagnie fabriquant des avions. Après avoir identifié les agents, nous identifions l'ensemble des tâches nécessitant la formation de groupes d'agents pour être exécutées. Nous obtenons le schéma suivant (cf. figure 4) décrivant la succession des activités ou tâches complexes. 
La formation de coalitions dans les chaînes logistiques revient essentiellement aux situations où il faut gérer des imprévus comme la modification d'une commande, les ressources qui tombent en panne, l'indisponibilité de certaines entités... A titre d'exemple, imaginons le scénario suivant :

"L'usine de Toulouse vient de recevoir une commande d'une compagnie du Moyen-Orient de 5 avions pour la fin de l'année prochaine. Après une étude complète du dossier, l'usine a constaté un manque en ressources pour satisfaire cette demande dans les délais. Elle décide alors de faire appel à d'autres entités capables de l'aider pour accomplir sa mission. »

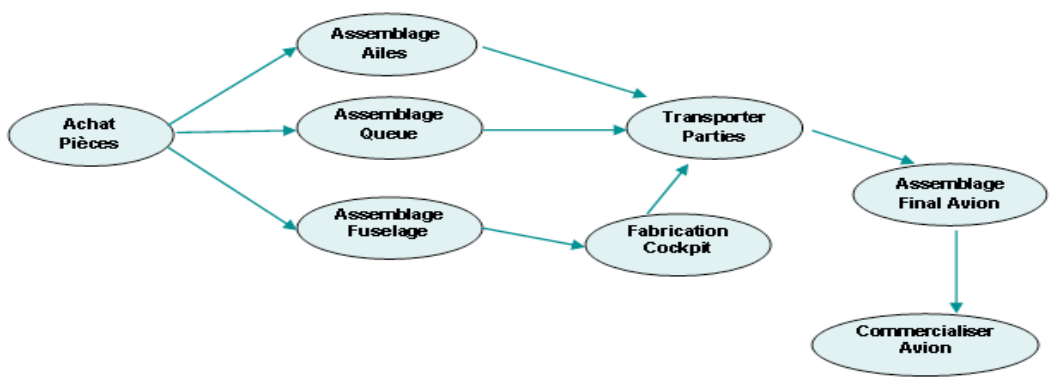

Figure 4. Exemple de succession d'activités de la chaîne logistique

En reprenant notre modélisation, l'usine de Toulouse qui est représentée par l'agent $a_{l}$ a identifié 3 agents potentiels qui pourraient l'aider à atteindre ses objectifs. Les agents à considérer lors du processus de formation de coalitions sont :

- $a_{1}$ : usine de production de Toulouse qui assure l'assemblage final et d'autres parties du fuselage.

- $a_{2}$ : usine de production de Grande- Bretagne qui fabrique les ailes.

- $a_{3}$ : usine de production d'Allemagne qui réalise les tronçons du fuselage.

- $a_{7}$ : usine de production concurrente en Chine qui fabrique les ailes, (cette entité est une entité externe).

A la suite de la commande de 5 avions à livrer avant la fin de l'année en cours, l'usine d'assemblage final lance une proposition de formation de coalitions avec d'autres entités pour atteindre ce but. Cette usine $a_{1}$ se met alors dans le rôle d'initiateur afin de former une coalition pour la tâche «assemblage final Avion ». Par hypothèse, chaque agent détient les informations nécessaires sur les compétences des autres agents et sur l'environnement dans lequel ils opèrent. Ces informations sont prédéfinies par le concepteur lors de l'implémentation du mécanisme au même titre que la définition des critères et leurs poids.

Reprenons l'exemple précédent avec les deux critères suivants :

- $p_{1}$ : délai de production : premier critère auquel on associe un poids individuel $\mu\left(p_{1}\right)=0.4$ 
- $p_{2}$ : distance : deuxième critère auquel on accorde un poids individuel de $\mu\left(p_{2}\right)=0.2$

- le poids collectif des deux critères est : $\mu\left(p_{1}, p_{2}\right)=1$

- Soit la matrice de préférences suivante : $\prod_{a_{1}}^{2}=\left(\begin{array}{cccc}a_{1} & a_{2} & a_{3} & a_{7} \\ 0 & 0.12 & 0.14 & 0.18 \\ 0 & 0.18 & 0.16 & 0.10\end{array}\right)$

- On peut noter, d'après les poids ci-dessus, que l'agent $a_{l}$ donne de l'importance au critère «délai de production» plutôt qu'au critère «distance ». Pour calculer sa préférence multicritère (vecteur), il utilise l'intégrale de Choquet comme opérateur d'agrégation (Grabisch, 1996), (Aknine et al. 2004). Il obtient :

$$
\prod_{a_{1}}=(0,0.132,0.144,0.132)
$$

Ce résultat est obtenu ainsi : $[(0.18-0.12) * 0.4+(0.12-0) * 1=0.144]$. De même, chacun des agents sollicités (ici, $a_{2}, a_{3}, a_{7}$ ) calcule son modèle de préférences et l'envoie à l'agent initiateur. Nous obtenons ainsi les vecteurs de préférences suivants :

$\prod_{a_{2}}=(0.148,0,0.157,0.11) \prod_{a_{3}}=(0.172,0.155,0,0.128) \prod_{a_{7}}=(0.163,0.138,0.146,0)$

L'agent initiateur passe alors au calcul de l'indice d'attraction unilatérale. Pour effectuer ce calcul, il doit disposer des $\mu\left(A_{j}\right)$ : poids individuels et collectifs des agents (cf. tableau 1.). Considérons les poids suivants :

\begin{tabular}{|c|c|c|c|}
\hline$\mu\left(a_{1}\right)=0.45$ & $\mu\left(a_{1}, a_{2}\right)=0.71$ & $\mu\left(a_{2}, a_{7}\right)=0.64$ & $\mu\left(a_{2}, a_{3}, a_{7}\right)=0.88$ \\
\hline$\mu\left(a_{2}\right)=0.25$ & $\mu\left(a_{1}, a_{3}\right)=0.63$ & $\mu\left(a_{3}, a_{7}\right)=0.79$ & $\mu\left(a_{1}, a_{2}, a_{3}, a_{7}\right)=1$ \\
\hline$\mu\left(a_{3}\right)=0.31$ & $\mu\left(a_{1}, a_{7}\right)=0.52$ & $\mu\left(a_{1}, a_{2}, a_{3}\right)=0.90$ & \\
\hline$\mu\left(a_{7}\right)=0.18$ & $\mu\left(a_{2}, a_{3}\right)=0.58$ & $\mu\left(a_{1}, a_{2}, a_{7}\right)=0.87$ & \\
\hline
\end{tabular}

Tableau 1. Poids individuels et collectifs des agents

Pour déterminer cet indice, cet agent classe par ordre décroissant les valeurs des préférences de l'agent $a_{i}$ avec tous les autres agents, par exemple pour $a_{1}$ nous obtenons les valeurs suivantes : $\left(x_{31}=0.172, x_{71}=0.163, x_{21}=0.148, x_{11}=0\right)$

$\mathbf{U}-\operatorname{Att}\left(\boldsymbol{a}_{1}\right)=(0.172-0.163) * 0.31+(0.163-0.148) * 0.79+(0.148-0) * 1=\mathbf{0 . 1 6 2}$

De même, pour les autres agents nous obtenons :

\begin{tabular}{|l|l|l|}
\hline U-Att $\left(a_{2}\right)=0.142$ & U-Att $\left(a_{3}\right)=0.148$ & U-Att $\left(a_{7}\right)=0.123$ \\
\hline
\end{tabular}

L'initiateur est celui qui a la valeur la plus élevée, i.e. $a_{l}$, il se met dans le rôle d'initiateur et calcule l'indice d'attraction bilatérale pour déterminer les autres agents à intégrer dans sa coalition. Le calcul est obtenu ainsi:

\begin{tabular}{|l|l|l|}
\hline B-Att $\left(a_{2}, C_{1}\right)=0.019$ & B-Att $\left(a_{3}, C_{1}\right)=0.024$ & B-Att $\left(a_{7}, C_{l}\right)=0.021$ \\
\hline
\end{tabular}

Exemple de calcul : B-Att $\left(a_{2}, C_{1}\right)=x_{12} * x_{21}=0.132 * 0.148=0.019$ 
L'agent $a_{3}$ possède la meilleure attraction pour la coalition que souhaite former l'agent $a_{1}$. Ce dernier lui envoie un message pour lui proposer d'intégrer sa coalition. De la même façon, $a_{3}$ commence son processus d'analyse de la proposition, en particulier, il examine la faisabilité de sa participation à la tâche concernée et ce en consultant la disponibilité de ses ressource et le coût engendré. Si ces deux conditions sont réunies, il confirme à l'initiateur qu'il souhaite participer à cette coalition. L'initiateur teste alors si son objectif peut être atteint par cette coalition, si c'est le cas, il arrête de contacter d'autres agents. Sinon il passe à l'agent ayant la valeur maximale suivante.

L'agent $a_{3}$ analyse la proposition de coalition pour vérifier si elle l'intéresse, il va par conséquent consulter ses compétences et surtout vérifier s'il dispose des ressources nécessaires à l'accomplissement de cette tâche. Ayant la liste des tâches qui précèdent cette dernière (ici la réalisation des tronçons de l'avion), il va examiner, par exemple:

- La disponibilité des matières premières en vérifiant les niveaux des stocks.

- La disponibilité des ressources en machines et en main d'œuvre.

- La planification du processus de production.

A chaque tâche, il calcule le coût correspondant noté dans notre algorithme par :

$$
c_{t_{s}}=\sum_{\left(t_{l}, t_{s}\right) \in E} c_{t_{l}} .
$$

A partir de cette analyse, l'agent sollicité décide s'il peut et/ou veut participer à cette coalition. Après décision, il envoie un message à l'initiateur, qui à son tour en fonction de cette réponse, envoie des messages à tout le reste des agents sollicités pour annuler ou pour confirmer cette coalition.

\subsection{Formalisation du protocole d'interaction entre agents :}

Tous les agents du système communiquent via des messages et suivent des règles prédéfinies dans leur protocole. Nous décrivons aussi bien la structure des messages ainsi que les règles du protocole. Un message est représenté par le vecteur suivant :

\section{Msg = <MsgType, SenderID, ReceiverID, Content $>$,}

Le champ «Content » comprend toutes les informations sur la coalition comme :

- La ou les tâches pour les quelles on souhaite former un groupe d'agents

- Les agents sollicités

- Les agents acceptés

- Les agents confirmés

- Les délais

Chaque message est d'un type spécifique : Réponse / Proposition / Confirmation

Chaque agent détient quatre listes de messages qui sont les suivantes : 
- proposedCoalitions : liste des coalitions proposées

- acceptedCoalitions : liste des coalitions acceptées (temporairement)

- refusedCoalition : liste des propositions qui ont été refusées

- confirmedCoalition : coalition confirmée (une seule)

En considérant les primitives suivantes (cf. tableau 2), le protocole d'interaction est représenté par deux diagrammes d'états : le premier décrivant le comportement de l'agent initiateur (I) et le deuxième décrivant le comportement des agents sollicités (S). A titre d'exemple, le premier protocole est présenté dans la figure 5.

\begin{tabular}{|c|c|l|}
\hline Primitive & De $\rightarrow$ Vers & \multicolumn{1}{|c|}{ Description } \\
\hline$(1,0)$ & $\mathrm{I} \rightarrow \mathrm{S}$ & Proposition \\
\hline$(2,1)$ & $\mathrm{S} \rightarrow \mathrm{I}$ & Acceptation Proposition \\
$(2,2)$ & $\mathrm{S} \rightarrow \mathrm{I}$ & Refus Proposition \\
$(2,3)$ & $\mathrm{S} \rightarrow \mathrm{I}$ & Confirmation Proposition \\
\hline$(3,1)$ & $\mathrm{I} \rightarrow \mathrm{S}$ & Demande Confirmation \\
$(3,2)$ & $\mathrm{I} \rightarrow \mathrm{S}$ & Annulation Proposition \\
\hline$(4,1)$ & $\mathrm{S} \rightarrow \mathrm{I}$ & Confirmation \\
$(4,2)$ & $\mathrm{S} \rightarrow \mathrm{I}$ & Annulation \\
\hline$(5,1)$ & $\mathrm{I} \rightarrow \mathrm{S}$ & Confirmation Coalition \\
$(5,2)$ & $\mathrm{I} \rightarrow \mathrm{S}$ & Annulation Coalition \\
\hline
\end{tabular}

Tableau 2. Les primitives du protocole d'interaction

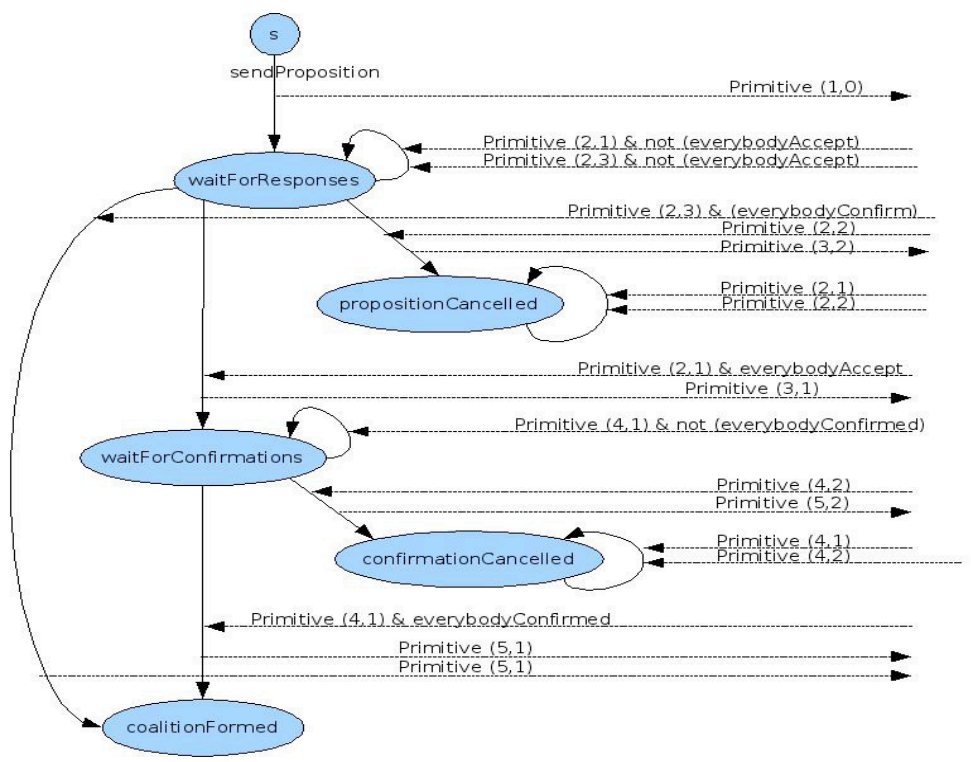

Figure 5. Comportement de l'initiateur de la formation de coalitions 


\section{Conclusion}

Dans cet article, nous avons montré que l'approche multi-agents est bien adaptée à la modélisation de la gestion d'une chaîne logistique. Dans le but d'améliorer et d'optimiser la gestion de la chaîne logistique, les agents doivent se coordonner pour réaliser des tâches communes. Nous proposons une méthode de coordination distribuée par formation de coalitions entre agents que nous empruntons au domaine des systèmes multi-agents. Etant donné que, dans les chaînes logistiques, les entités apparaissent et disparaissent dynamiquement, la modélisation agents permet de simuler des systèmes ouverts ce qui garantit la flexibilité, l'efficacité et l'évolutivité de ces systèmes.

Nous avons, dans un deuxième temps, présenté la structure des messages échangés et le protocole de communication entre agents sur lequel s'appuie l'algorithme de formation de coalitions. Nous avons illustré nos propositions par un exemple de chaîne logistique pris dans le domaine industriel. Un prototype multiagents mettant en œuvre notre algorithme de coordination et le protocole d'interaction est en cours de développement et de tests.

Comme perspectives de recherche, plusieurs hypothèses faites au cours de la modélisation peuvent être étudiées : 1) une fois la formation de coalitions choisie comme méthode de coordination, le choix et la définition du protocole correspondant restent problématiques. Ce choix est en réalité très dépendant du type de problème étudié c'est-à-dire des paramètres à prendre en considération comme par exemple le fait que les agents ont ou non le même objectif voire la même fonction d'utilité, ou le fait qu'ils se font ou non confiance pour pouvoir s'échanger leurs connaissances... tous ces paramètres peuvent générer des protocoles totalement différents qui doivent être testés, 2) le protocole proposé repose sur plusieurs principes qui peuvent ne pas être à chaque fois adaptés à la réalité industrielle en particulier dans le cas d'une concurrence entre les différents partenaires d'une chaîne logistique. Ils peuvent aussi avoir des objectifs radicalement opposés comme dans la relation fournisseur-producteur, tout dépendra alors du déroulement de la négociation qu'il faudra modéliser.

\section{Bibliographie}

Aknine S., Pinson S. et Shakun M. (2004), "A Multi-Agent Coalition Formation Based on Preference Models", International Journal Group and Decision and Negotiation, Kluwer. Vol $13 \mathrm{~N}^{\circ} 6$, p 513-538.

Barbuceanu M., Fox M. S. (1996), "The Architecture of an Agent Building Shell", in Intelligent Agents II , Wooldridge M., Muller J. P. and Tambe M.(Eds.) Lecture Notes in Artificial Intelligence, Springer-Verlag, Berlin, Vol 1037 p 235-250

Beamon B. M. (1998), "Supply Chain design and analysis: Models and Methods", The International Journal of Production Economics, $\mathrm{N}^{\circ} 55 \mathrm{p} 281-294$.

Chaib-draa B., Asselin, F. (2002), "Towards a protocol for the formation of coalitions of buyers", Proceedings of the 5th Intenational Conference on Electronic Commerce Research. 
Davis R. et Smith R. G. (1988), "Negotiation as a Metaphor for Distributed Problem Solving", Artificial Intelligence (20) 1; p 63-109.

Dodd C. et Kumara S.R.T. (2001). "A distributed multi-agent model for value nets”. IEA/AIE 2001, pages 718-727.

Fox M. S., Barbuceanu M. (1996), "Coordinating multiple agents in the Supply Chain", Proceedings of Fifth Workshop on Enabling Technologies: Infrastructure for Collaborative Enterprises, Stanford, CA, IEEE Computer Society Press, pp 134-142.

Fox M. S., Barbuceanu M. et Teigen, R. (2000), "Agent-Oriented Supply-Chain Management", The International Journal of Flexible Manufacturing Systems, 12 p 165188.

Giard V., Balin, S. (2007) "Problèmes méthodologiques posés par la simulation de processus de production de services”, Journal Européen des Systèmes Automatisés (APII-JESA) 41(9-10), 2007.

Grabisch, M. (1996) "The Application of Fuzzy Integrals in Multi-criteria Decision Making". European Journal of Operational Research, Vol. 89.

Hahndel S., Fuchs F. et Levi P. (1994) "Distributed Negotiation-Based Task Planning for a Flexible Manufacturing Environment". In Proceedings of the 6th European Workshop on Modeling Autonomous Agents in Multi-Agent World, p 147- 158. Odense, Denmark.

Huget M. P. (2002). "An application of Agent UML to Supply Chain Management". In Proceedings of Agent Oriented Information System (AOIS-02). Paolo Giorgini and Yves Lespérance and Gerd Wagner and Eric Yu (eds.), Bologne, Italie.

Kok, A. G. et Graves, S. C. (2003), "Supply Chain Management: Design, Coordination and Operation", Handbooks in Operation Research and Management Science, Elsevier Vol. $11 \mathrm{p} 1-16$.

Nfaoui H. et Ozrout Y. (2006) "SCM, AUML: un modèle d'agents pour la simulation proactive et l'aide à la décision dans le Supply Chain", Conférence Majestic 2006. web.univ-ubs.fr/lester/www-lester/Evenements/Majecstic/papers.

Parunak, H.V.D. (1996). "Applications of distributed artificial intelligence in industry". In O'Hare, G. M. P. and Jennings, N. R., editors, Foundations of Distributed Artificial Intelligence, p 71-76. Ed. John Wiley \& Sons.

Parunak H. V. D. et VanderBok R. (1998), “Modeling the extended Supply Networks”, In ISA-Tech'98 (Houston), Industrial Technology Institute.

Shehory O. et Kraus S. (1996), "Formation of overlapping coalitions for Precedence-ordered Task Execution among Autonomous Agents"; In proceedings of ICMAS-96, p 330-337, Kyoto, Japan.

Shehory O. et Kraus S. (1998), "Methods for Task Allocation via Agent Coalition Formation"; Artificial Intelligence Journal, Elsevier Science Vol. 101 (1-2) p 165-200.

Swaminathan J.M, Smith S.F et Sadeh N.M (1998), "Modeling Supply Chain Dynamics: A Multi Agent Approach”; Decision Sciences Journal, Vol. 29, N 2 p 607-632. 